\title{
Origins of Knowledge: Insights from Precocial Species
}

\author{
Elisabetta Versace * and Giorgio Vallortigara* \\ Animal Cognition and Neuroscience Laboratory, Center for Mind/Brain Sciences, University of Trento, Rovereto, Italy
}

Behavioral responses are influenced by knowledge acquired during the lifetime of an individual and by predispositions transmitted across generations. Establishing the origin of knowledge and the role of the unlearned component is a challenging task, given that both learned and unlearned knowledge can orient perception, learning, and the encoding of environmental features since the first stages of life. Ethical and practical issues constrain the investigation of unlearned knowledge in altricial species, including human beings. On the contrary, precocial animals can be tested on a wide range of tasks and capabilities immediately after birth and in controlled rearing conditions. Insects and precocial avian species are very convenient models to dissect the knowledge systems that enable young individuals to cope with their environment in the absence of specific previous experience. We present the state of the art of research on the origins of knowledge that comes from different models and disciplines. Insects have been mainly used to investigate unlearned sensory preferences and prepared learning mechanisms. The relative simplicity of the neural system and fast life cycle of insects make them ideal models to investigate the neural circuitry and evolutionary dynamics of unlearned traits.

OPEN ACCESS

Edited by:

Caroline Helen Brennan, Queen Mary University of London,

UK

Reviewed by:

Maria Dolores De Hevia, Centre National de la Recherche

Scientifique, France Matthew O. Parker, University of Portsmouth, UK

*Correspondence: Elisabetta Versace elisabetta.versace@unitn.it; Giorgio Vallortigara giorgio.vallortigara@unitn.it

Received: 09 April 2015 Accepted: 20 November 2015 Published: 09 December 2015

Citation: Versace $E$ and Vallortigara G (2015) Origins of Knowledge: Insights from Precocial Species.

Front. Behav. Neurosci. 9:338. doi: 10.3389/fnbeh.2015.00338 Among avian species, chicks of the domestic fowl have been the focus of many studies, and showed to possess unlearned knowledge in the sensory, physical, spatial, numerical and social domains. Solid evidence shows the existence of unlearned knowledge in different domains in several species, from sensory and social preferences to the leftright representation of the mental number line. We show how non-mammalian models of cognition, and in particular precocial species, can shed light into the adaptive value and evolutionary history of unlearned knowledge.

Keywords: origins of knowledge, precocial species, insects, avian species, Drosophila, Gallus gallus, domestic chicks

\section{INTRODUCTION}

Precocious knowledge can help naïve individuals in making correct predictions and deciding whether to approach or avoid an object and how to cope with a situation encountered for the first time.

Evidence of precocious knowledge has been documented in species with a short life span, when learning by trial and error could be too costly. For instance, in the absence of previous experience, fruit flies (Drosophila melanogaster) prefer to lay their eggs on fruit containing limonene, a behavior that helps them contrasting predation from parasitic wasps (Dweck et al., 2013). Examples of knowledge supported by little if any learning have been found also in species that live much longer than fruit flies, such as human beings and chicks of the domestic fowl (Gallus gallus), and in tasks that can involve less dramatic 
consequences in case of failure. Along with other species, young human beings know from the earliest stages of their life that a large object cannot be hidden behind a narrower occluder (Aguiar and Baillargeon, 1999, 2002; Chiandetti and Vallortigara, 2011b), that objects maintain their identity in spite of proximal changes on the retina (Wood and Wood, 2015), and continue to exist when occluded (Baillargeon et al., 1985; Regolin et al., 1995), that three dots are different from two (Rugani et al., 2009), $1 / 4$ is different from $3 / 4$ Rugani et al. (2014) and 4 is different from 12 (Izard et al., 2009), that an attractive face has two eyes and one mouth (Turati et al., 2002; Rosa-Salva et al., 2010), that cliffs are more dangerous than solid ground (Walk and Gibson, 1961).

The diverse examples of early knowledge can be conveniently grouped in two categories to help an interspecific comparison: (a) predispositions to attend or orient towards or away from particular stimuli (e.g., specific shapes (Fantz, 1957; Zanforlin and Vallortigara, 1985), colors (Hess and Gogel, 1954; Hess, 1956), and stimuli associated with social partners such as face-like stimuli (Johnson and Horn, 1988; Rosa-Salva et al., 2010), stimuli moving according to biological motion dynamics (Vallortigara et al., 2005; Vallortigara and Regolin, 2006; Simion et al., 2008) and (b) unconditioned assumptions/expectations about physical facts of the world that can in turn bias learning of certain stimuli or associations (e.g., constraints on learning (Garcia and Koelling, 1966; LoLordo, 1979) or preparedness for learning (Dunlap and Stephens, 2014) or help problem solving (e.g., object permanence (Regolin et al., 1995), physical reasoning about occluded objects (Chiandetti and Vallortigara, 2011b), use of geometrical information for spatial cognition and navigation (Tommasi et al., 2012).

In most of the cases, the origin of precocious knowledge and its evolutionary basis cannot be easily established. Knowledge available in the first stages of life can either be acquired through early specific stimulation-including embryonic experiences (Lecanuet and Schaal, 1996; Schaal et al., 2000; Graven and Browne, 2008; Harshaw and Lickliter, 2011)—or transmitted between generations, in the form of predispositions that orient perception, learning and other cognitive functions during the course of normal development. Precocial species, which are able to move and feed soon after birth and require little if any parental care, can have a great benefit from unlearned knowledge. Moreover, the life history of insect species who did not evolve parental care (Wong et al., 2013) gives them a privileged position for the investigation of unlearned knowledge. Insect species with no post-hatching parental care exhibit several unlearned abilities, such as phototaxis, thermotaxis and chemotaxis (e.g., Gong, 2012; Dubnau, 2014). In the aforementioned case of fruit flies and citrus preference, experimenters can control the environment to exclude a role of specific experience, try to identify the sensory-motor circuits that produce preferential choices, compare the ecology and oviposition choices of different species to investigate the adaptive value of a trait, or even run experimental evolution to investigate evolutionary responses at the behavioral and genetic level in a reasonable amount of time
(Schlötterer et al., 2015; Versace, 2015). While most of studies involving insects focused on sensory preferences and preparedness for learning/constraints on learning (e.g., Giurfa et al., 1995; Dweck et al., 2013; Dunlap and Stephens, 2014), more recently other cognitive domains such as unlearned spatial cognition and navigation abilities (Lee and Vallortigara, 2015) and general learning abilities (Mery and Kawecki, 2002) have been investigated.

In the case of youngster of altricial species-including human beings, which are dependent on parental care for a long time, the options to investigate the origins of knowledge are much more limited. For centuries philosophers and scientists (reviewed in Hess, 1973; Spelke and Newport, 1998; Spelke, 1998) have been asking to which extent our perceptions and reactions depend on predisposed mechanisms disengaged from specific experience (nativist hypotheses) or on the specific experiences we have been exposed to (empiricist hypotheses). In the last years new techniques have been developed to measure precocious reactions and sensitivity to a variety of stimuli in infants (reviewed in Streri et al., 2013), that range from behavioral (e.g., habituation/dishabituation procedures (Aslin, 2007) to neuroimaging approaches (e.g., near infrared spectroscopy (Bartocci et al., 2000) and magnetoencephalography Cheour et al. (2004)). In spite of this, the range of capabilities that can be investigated soon after birth is still limited by the reduced range of motor actions and immaturity of perceptual abilities in infants. ${ }^{1}$

Moreover, for ethical and practical reasons primate babies cannot be raised in completely controlled rearing conditions or be deprived of important stimuli such as light or social contact until the moment of test, as it is done in isolation experiments. $^{2}$ Wood and Wood (2015) have reviewed similar constraints in rodent species, for whom rearing in darkness prevents complete and normal maturation in the visual cortex, alters the development of GABAergic transmission, and cannot be raised from birth in controlled, lighted environments. In the light of the limitations that constrain research in altricial species, questions about the origin of unlearned knowledge might be more conveniently addressed using precocial species.

Avian precocial species (species that move around and feed on their own soon after hatching) such as young domestic chickens (Gallus gallus), goslings and ducklings have been used to investigate the existence and content of precocious knowledge in vertebrate species since the late nineteenth century (for a review, see Andrew, 1991). As for acoustic stimulation, Gottlieb (1968) showed that in ducks embryonic auditory experience with the own calls produces species-specific preferences for

\footnotetext{
${ }^{1}$ Similar limitations apply to other altricial species, as certain cichlid fish, that require an extended period of development before reaching a complete sensory-motor maturation and behavioral performance (Barlow, 2000). Fish species such as the zebrafish (Danio rerio), showed to be convenient models for the investigation of social cognition (Oliveira, 2013).

${ }^{2}$ This approach can clarify which input is not necessary for the development of a certain behavior, and not that no experience is not required for the development of a certain behavior, because a certain amount of experience is always present.
} 
acoustical stimuli. In this species the acoustic system is tuned in such a way that the little experience gained by the embryo is sufficient to orient subsequent approaching responses to conspecific calls (Gottlieb, 1965). Similar results have been found in bobwhite quail chicks (Lickliter and Stoumbos, 1991). Prenatal learning of sounds has been observed in altricial species, including human beings (for a review, see Lecanuet and Schaal, 1996). As for visual stimuli, a wide range of experiments has identified preferences of naive chicks to approach specific colors (Schaefer and Hess, 1951; Hess and Gogel, 1954), size (Schulman et al., 1970), shapes (Fantz, 1957; Zanforlin and Vallortigara, 1985), as well as configurations of static (e.g., face-like stimuli (Rosa-Salva et al., 2010) and dynamic stimuli (e.g., biological motion (Vallortigara et al., 2005), self-propulsion (Mascalzoni et al., 2010). The preferential orientation towards face-like stimuli has been attributed to the so-called CONSPEC system (Morton and Johnson, 1991). This mechanism specifies information on the general features of social partners and care-givers, as opposed to the socalled CONLERN system, devoted to learning the particular visual characteristics of specific individuals. Consistent with this hypothesis is the fact that precocial avian species which in the wild develop an attachment to the mother, share the preference for cues associated with social partners and care-givers with newborns of our species (e.g., Morton and Johnson, 1991; Simion et al., 2008). The co-occurrence of early preferences for face-like stimuli, biological motion and self-propelled objects suggests that social species might be endowed with a core knowledge system dedicated to social stimuli (Spelke and Kinzler, 2007; Vallortigara, 2012b).This suggestive hypothesis could be validated by investigating the differences in reactions to social stimuli between breeds and species that require a different degree of parental care soon after birth. Reptiles such as tortoises, which require no post-hatching parental care, are a good model system to understand the adaptive role and evolution of social coreknowledge mechanisms. While it has been showed that adult tortoises have the capability to follow the gaze of conspecifics (Wilkinson et al., 2010), little is known on their early social capabilities.

\section{PREDISPOSITIONS FOR ATTRACTIVE AND AVERSIVE STIMULI}

\section{Sensory Predispositions}

Nativist theories have found support in the domain of sensory development (e.g., Walk et al., 1957; Walk and Gibson, 1961; Blakemore and Van Sluyters, 1975; Li and Liberles, 2015). The olfactory system of Drosophila is a good model for probing these theories. Compared to other sensory modalities, olfactory circuits require few connections to convert sensory inputs into behavioral responses [see $\mathrm{CO}_{2}$ avoidance (Suh et al., 2004)], and pheromone detection and courtship behavior (Dulac and Wagner, 2006; Ruta et al., 2010). Fruit flies exhibit consistent unlearned attraction and repulsion for food- and oviposition-related odors (Ruebenbauer et al., 2008; Semmelhack and Wang, 2009; Dweck et al., 2013; Min et al., 2013; Ronderos et al., 2014; Versace and
Reisenberger, 2015) but these behavioral responses are not fixed. Peripheral (Krishnan et al., 1999, 2008; Root et al., 2011) as well as central circuits (Semmelhack and Wang, 2009) encode the valence of stimuli depending on odor-concentration-higher concentrations can recruit additional receptors, change central representations and modify the perceived valence (Semmelhack and Wang, 2009) - and the internal physiological state of the individual (e.g., circadian changes in olfactory sensory neurons have been observed (Krishnan et al., 1999, 2008); starved flies are differently responsive to odors compared to fed flies (Root et al., 2011). Hence in fruit flies attraction to food odors is state-dependent, both at the peripheral and central level.

The relative simplicity of the courtship behavior circuits in fruit flies makes the investigation of the perception-action circuitry much more feasible than in vertebrate species (Datta et al., 2008). While at the peripheral level exposure to the male emitted pheromone cis-vaccenyl acetate produces similar responses in males and females, the behavioral responses are completely different in the two sexes: females increase receptivity to courting males, whereas males show an increase of aggression towards males and suppression of courtship. These sexually dimorphic differences are mediated by anatomical and functional differences in the central nervous system. In particular, specific regions of the lateral horn receive projections from glomeruli linked to odor attraction and aversion, suggesting that valence is spatially encoded in this structure (Min et al., 2013). The link between lateral horn neurons and behavioral responses has not yet been entirely clarified but by tracing the pheromone-response circuit researchers have revealed lateral horn neurons with sexually dimorphic projections, including projections to male descending neurons, that enter the ventral nerve cord, which in turn controls movement (Ruta et al., 2010).

Unlearned preferences are widespread in the visual modality too. To make a few examples, fruit flies show phototactic responses (Gao et al., 2008; Yamaguchi et al., 2010), several insects have color preferences for flowers (Giurfa et al., 1995; Lunau and Maier, 1995; Gumbert, 2000) and naïve chicks display visual preferences for pecking objects (Hess and Gogel, 1954; Fantz, 1957) and for imprinting objects (Schaefer and Hess, 1951; Kovach, 1983, 1990; see also Rosa Salva et al., 2015 for a review about preferences for imprinting objects). The investigation of visual unlearned preferences in human infants has a long tradition: already in the Sixties Fantz (1963) showed a greater responsiveness to patterns than to hues. Recent work with children (8-16 years old) who gained sight after earlyonset blindness, provides evidence of unlearned susceptibility to the Ponzo and Müller-Lyer illusions immediately after the onset of sight (Gandhi et al., 2015). This evidence points at the role of unlearned knowledge also in our species. We will discuss preferences for configurations of stimuli in human and non-human newborns in the next section. The complexity of the visual circuitry makes the investigation of the perception-action link more difficult than in the case of olfactory perception. 


\section{Structural Features and Structured Events}

Beside sensory attraction and avoidance reactions for perceptual stimuli, predispositions to orient towards or avoid configurations of stimuli since the early stages of life have been identified in different species.

In the visual domain the case of reactions to face-like stimuli is paradigmatic. Stimuli that reproduce the configuration of faces, with two centered eye-like spots in the upper part and one centered mouth-like spot in the lower part, can trigger attraction responses. Few hours after birth, human newborns follow more effectively the movement of schematic face-like stimuli compared to scrambled faces, blank stimuli, upside-down face-like stimuli and top heavy stimuli (Goren et al., 1975; Johnson et al., 1991), and in the absence of visual experience with faces, naïve newly hatched chickens show an analog preference to approach facelike stimuli (Johnson and Horn, 1988; Rosa-Salva et al., 2010). This predisposition, shared between social species, has been attributed to a mechanism of preferential tuning towards caregivers, namely the CONSPEC mechanism (see Morton and Johnson, 1991). Eye-like stimuli can also elicit anti-predatory responses such as tonic immobility in chickens (Gallup and Nash, 1971; Gagliardi et al., 1976) and have been suggested to produce avoidance in insectivorous birds (Janzen et al., 2010; Hossie and Sherratt, 2013), although salience can be the major drive of this effect (Stevens et al., 2008). The neural circuits that mediate unlearned responses to faces and eye-like stimuli have not been completely identified, but some evidence suggests that in our species the initial attraction for faces might be mediated by subcortical areas (e.g., superior colliculus or homologous areas (Rosa Salva et al., 2015), while subsequent stages rely more on cortical areas (Johnson et al., 2014).

Precocial biases for biological vs. rigid motion (Vallortigara et al., 2005; Simion et al., 2008) and for self-propelled objects (Mascalzoni et al., 2010, 2013) can produce a preferential orientation towards structural and configurational patterns associated with animate entities. When points of light are attached to the joints of a moving animal kept in darkness, the animation conveys information about the animal's activity that suggests the idea of biological motion Johansson (1973). Inexperienced newly hatched chicks (Vallortigara et al., 2005), as well as human newborns (Simion et al., 2008), show a spontaneous preference to approach/preferentially look at stimuli that move according to semi-rigid trajectories of a walking animal (biological motion) vs. the same animal rotating on the central axis in a rigid fashion (see also Rugani et al., 2015a). Naïve chicks (Mascalzoni et al., 2010) and human newborns (Di Giorgio et al., 2014) prefer to approach/fixate objects that exhibit self-produced motion compared to objects that move only after the contact with a moving object, and 5-month-old infants understand that objects can start to move only as a result of contact with another moving object, unless they are provided with inner energy that permits self-produced motion (Luo and Baillargeon, 2005).

In the acoustic modality, it has been shown that 1-3 days old newborn babies show differential hemispheric activation during the presentation of consonant $v s$. dissonant tones
(Perani et al., 2010), suggesting early functional specialization for specific sounds. Moreover, 2 and 4-month old infants prefer to listen to consonant over dissonant intervals (Trainor et al., 2002). These preferences could be acquired not only through early exposure to music but also through embryonic experience, given the acoustic sensitivity of the fetus (for a review, see Hepper and Shahidullah, 1994). In the acoustic modality, Chiandetti and Vallortigara (2011a) have shown a precocial preference of newly hatched chicks for consonant $v s$. dissonant intervals, a phenomenon that could guide newly hatched chicks to attend to (and imprint on) animate rather than inanimate objects, given that the harmonic relationships between frequency components is associated with prominent features of sounds in natural environments.

These findings about unlearned preferences for structured stimuli that correlate with the presence of animate objects (facelike stimuli, biological motion, self-propelled objects, potentially consonant intervals) suggest that different vertebrate species may share a predisposed neural mechanism to detect animate objects (Vallortigara, 2012b). A core knowledge system for social cognition could have evolved to help newborns of social species in the interaction with their care-givers. Comparative work with precocial non-social species can help clarifying this issue.

\section{PREDISPOSITIONS FOR LEARNING AND UNCONDITIONED ASSUMPTIONS}

\section{Predispositions for Preferential/Faster Learning of Specific Stimuli or Associations}

Since the seminal work conducted on rats, which showed an easier association of audio-visual stimuli with electric shock and gustatory stimuli with gut sickness (Krechevsky, 1933; Garcia and Koelling, 1966), it is known that some predispositions exist that can ease or hinder the acquisition of specific associations. Other examples of species-specific biases, known as constraints on learning or preparedness for learning, include the preferential use of the "win-shift" over "win-stay" strategies in birds during foraging (Gill and Wolf, 1977; Kamil, 1978; Cole et al., 1982). The convenience of insects as model species has recently emerged in this field of research. Taking advantage of the learning abilities and fast life cycle of $D$. melanogaster, Dunlap and Stephens (2009, 2014) have used fruit flies to show the role of the environment in shaping learning mechanisms. By pairing an aversive taste contingency with color or with odor for dozens of subsequent generations, the authors found empirical support to the hypothesis that the reliability of associations encountered during the evolutionary history shapes the learning abilities of a species (Dunlap and Stephens, 2014). Experimental evolution on fruit flies has showed also the possibility to select for enhanced associative learning abilities in the olfactory domain (Mery and Kawecki, 2002). Organisms with a fast life cycle are extremely important to understand the evolutionary basis of cognitive mechanisms, including unlearned predispositions, at the behavioral and genetic level (Versace, 2015). 


\section{Unconditioned Assumption/Expectations}

Few months after birth, human babies understand that objects continue to exist even when they are no longer visible (object permanence, see Baillargeon et al., 1985), reason about the physical properties of objects such as height, size and solidity (Aguiar and Baillargeon, 1999, 2002), encode the location of objects (Newcombe et al., 1999) and encode causal relationships (Leslie and Keeble, 1987; Mascalzoni et al., 2013). This evidence though is compatible both with precocial learning and unlearned capabilities (or maturation of unlearned capabilities).

Increasing evidence collected with precocial species is clarifying to which extent unlearned knowledge about physical facts of the world can help animals to cope with the environment and in problem solving since the earliest stages of life. In spite of continuous changes in lightning, distance and point of view, different species have no difficulties in recognizing an object. Young chicks of nidifugal species develop an attachment and following response, known as filial imprinting (reviewed in Bateson, 1991), for the first conspicuous object encountered in their life. In nature this object is usually the mother hen. To understand whether chicks require experience with multiple appearances of the same object to produce invariant object recognition of the imprinting object, Wood and Wood (2015) have reared newly hatched chicks in a controlled imprinting environment with partial and impoverished experience with an imprinting object. Following exposure with only three views of a single object, chicks could build an invariant representation of it.

Object permanence has been investigated in young chicks that had no previous experience with occluded objects. By using imprinting objects disappearing behind occluders of different size, Chiandetti and Vallortigara (2011b) showed object permanence abilities in the absence of previous experience and spontaneous physical reasoning about occluded objects. In fact chicks expected that imprinting objects had disappeared more likely behind occluders bigger than their size than behind occluders smaller than their size.

Orientation, navigation and location memory rely on environmental cues such as the geometric features of the environment (Vallortigara et al., 2009). Newly hatched chicks are able to encode and use geometric information to search for a disappeared imprinting object in the absence of previous experience (Chiandetti et al., 2014). Fish (Archocentrus nigrofasciatus, Brown et al., 2007) as well as chicks of the domestic fowl (Chiandetti and Vallortigara, 2008) reared in home cages of different geometric shapes are equally capable of learning and performing navigational tasks using geometric information. These findings suggest that the effective use of geometric information does not require specific metric and geometrical experience (Chiandetti et al., 2014). Recent studies on bumble bees have shown that spontaneous use of geometrical features, boundaries and local environmental features (Lee and Vallortigara, 2015) can guide the untrained spatial behavior in these insects. Behavioral studies have just started to show the potential of insect models in the study of unlearned knowledge used in "high-level" cognitive abilities (Vallortigara et al., 1999).
Another striking example of precocious and untrained abilities is numerical cognition. Numerical abilities have been found in human infants (Xu et al., 2005; Libertus et al., 2009; Libertus and Brannon, 2010) and even newborns (Izard et al., 2009; Coubart et al., 2014; De Hevia et al., 2014). These studies offer good evidence for abstract numerical representations at the start of postnatal experience and parallel similar evidence obtained in newborn chicks (e.g., Rugani et al., 2014; and see also comments in Brugger, 2015; Drucker and Brannon, 2015). Infants habituated to pictures of the same number of objects (e.g., 8 dots) looked longer when presented with arrays of a new numerical value controlled for continuous variables (e.g., 16 dots; Xu, 2003; Brannon et al., 2004; Xu et al., 2005), thus showing an early sense of number/numerosity. Individual differences in this task remain stable during infancy (Libertus and Brannon, 2010; De Hevia and Spelke, 2010) and are a good predictor for subsequent mathematical abilities in childhood (Starr et al., 2013). Similarly to other domains, the learned/unlearned origin of this knowledge can be hardly established in human beings. Young chicks can be used to investigate precocial protomathematical cognition based on their need of proximity to social partners to reach an optimal temperature and avoid predators since the early stages of life. After imprinting, chicks treat imprinting objects as social partners and prefer to approach sets with larger number of imprinting objects, showing their untrained capability to distinguish between sets of different numerosities. This behavior does not simply reflect a general preference for larger sets or for larger extent of continuous features, since when presented with novel objects chicks approach the group with the same numerousness as in the original imprinting condition (Rugani et al., 2010) when continuous features are controlled. In chicks, even basic arithmetic skills are available at birth and in the absence of formal training, as shown by the capability to approach the larger set of imprinting objects that results after displacement (corresponding to addition or subtraction) of occluded imprinting objects (Rugani et al., 2009). In these experiments after imprinting the chicks saw a set of four and a set of one imprinting objects disappearing behind a screen. Subsequently chicks were shown imprinting objects moving between the screens. When left free to move, they chose to approach the screen that currently hide the largest number of objects, thus showing proto arithmetical capabilities to sum and subtract numerosities. Experiments run on numerical encoding in this species have clarified an untrained left-right representation of the ordinal value of numbers, suggesting that the left-right representation of the mental number line in human beings can reflect more a biological predisposition than mere cultural conditioning (Rugani et al., 2015b).

\section{CONCLUSIONS}

In the absence of previous specific experience, unlearned knowledge can guide behavioral responses of approach and avoidance, and provide cues to problem solving. Establishing 
whether a response is guided by previous learning or it is rather spontaneously determined requires controlled rearing conditions until the moment of the experiment. By collecting evidence from disciplines as different as evolutionary biology, animal cognition and developmental psychology we have showed how different models and approaches can help clarifying the origins of precocious knowledge. When young individuals of altricial species are too immature to be tested before the acquisition of significant experience in the domain of interest, often precocial species are convenient models. This does not of course deny the existence of unlearned knowledge in human beings. There is indeed some straightforward evidence of this, as shown for example by some of the work with human newborns that provided evidence of abstract number at the start of postnatal life (see "Unconditioned Assumption/Expectations" Section). For instance, Izard et al. (2009) showed that newborn infants spontaneously associate stationary, visual-spatial arrays of 4-18 objects with auditory sequences of events on the basis of number. Here it seems unlikely (though perhaps it cannot be completely excluded) that newborns before the tests have had opportunities and time enough to associate, on the basis of their early minimal multimodal perceptions, visual and auditory stimuli which were time-locked and then to generalize the link to non time-locked stimulation. Besides, it is difficult to imagine how such a link can be formed without a predisposed abstract notion of number. Yet, in other cases it is virtually impossible to prevent human newborns from having even minimal visual experiences, and thus early learning, that can easily account for their precocious abilities (e.g., exposure to faces for predisposition to face-like stimuli, exposure to extended visual surfaces in the spatial layout for predisposition to geometry and so on).

Insect of species that do not require post-hatching parental care, such as the well-studied model Drosophila melanogaster, can be easily controlled until the moment of test and investigated at the behavioral, neurobiological and genetic level. Moreover, the relative simplicity of the olfactory circuits in insects makes the investigation of neural mechanisms feasible, and genetic analysis has already shed light into the genetic determinants of olfactory behavior (Rollmann et al., 2010; Arya et al., 2015). Other research has shown the potential of phylogenetically distant models for biomedical research on human beings (Reiter et al., 2001). Moreover, fruit flies have a life cycle fast enough to be monitored during experimental evolution for changes in their predispositions (and underlying genetic changes (Schlötterer et al., 2015; Versace, 2015). When selected for different associations (color/flavor $v s$. odor/flavor) fruit flies changed their promptness in learning the association used during selected (Dunlap and Stephens, 2014), thus indicating the evolutionary basis of preparedness for learning.

Avian precocial species, such chicks of the domestic fowl, have been extensively used to investigate unlearned knowledge about configurations of cues as well as unconditioned expectations about facts of the world that can to cope with the environment. The precocial preference for stimuli associated with the presence of animate objects that has been found in both naive chicks and human newborns-e.g., face-like stimuli (Rosa-Salva et al.,
2010), biological motion (Vallortigara et al., 2005; Simion et al., 2008)-suggests that species that require post-birth parental care might rely on an adaptive social core knowledge system moulded by natural selection. This hypothesis can be validated through comparative research that investigates the responses of precocial species with no post-hatching parental care, such as Chelonia. This example clearly shows that the interest in non-mammalian species is theoretically important, and not only justified by ethical reasons (e.g., primate infants cannot be controlled-reared until they reach the maturation required for behavioral tests), or by practical convenience (e.g., precocial avian species can be easily manipulated until the moment of the test and possess developed sensory and motor capabilities soon after hatching). The evidence collected in the last decades (Vallortigara, 2012b; reviewed in Spelke and Kinzler, 2007; Vallortigara et al., 2009; Spelke et al., 2010; Vallortigara, 2015), with the notable examples of unlearned number (e.g., Rugani et al., 2009, 2014) and spatial cognition (e.g., Vallortigara et al., 2009; Lee and Vallortigara, 2015) show the continuity of the human mind with other species, including phylogenetically distant species. By comparing different breeds and species it will possible to shed light into the adaptive value and evolutionary history of unlearned knowledge, and to understand the connection between sensory or "low-level" processes and other cognitive abilities, such as the link between the number sense in infancy and mathematical abilities in childhood (Starr et al., 2013). Furthermore, behavioral tests developed on precocial species, and therefore well-validated for their independence from specific experiential effects (again, social, space and number cognition provide excellent examples, see for instance (Haun et al., 2010; Vallortigara, 2012a,b) may set the stage for the development of similar tests in species that are not precocial but offer on the other hand peculiar advantages for molecular and genetic analyses (zebrafish is an obvious example, for social cognition see for instance Oliveira, 2013), for space cognition (see Lee et al., 2013; for number cognition see Potrich et al., 2015), thus favoring the step to translational research.

Precocial species have proven to have high translational value for areas associated with neurocognitive disturbances and developmental disorders. Van der Voet et al. (2014) have recently reviewed the importance of Drosophila for translational research on dozens of early onset cognitive disorders, from Fragile X syndrome (the most extensively studied) to Autism spectrum disorders (e.g., Hahn et al., 2013 for impairment of social behaviors in mutant flies), highlighting the convenience of fruit flies to investigate gene function, determine primary origin of pathology and identifying specific genes involved, test hypotheses on in vivo models, acquire disease-relevant tissues, combine mutations in different genes to dissect molecular networks. Genetic and neurobiological approaches can be conveniently applied to fruit flies to study unlearned behaviors and clarify the role of environmental cues in eliciting specific responses, circuits and computations involved in preprogrammed behaviors (Manoli et al., 2006). Vertebrate nonmammalian species are also highly valuable translational models. For instance, besides being a classical model for epilepsy (see 
for instance Douaud et al., 2011 for recent studies) the domestic chick is a well-established model for the study of the cellular mechanisms of memory and potential therapies for dementia diseases (Mileusnic and Rose, 2010). Furthermore, studies on precocial social species have permitted the formulation of original hypothesis on some developmental disturbances such as autism. The so-called "social orienting hypothesis" assumes that unusual development of the social brain in individuals with autism spectrum disorders may be due to an alteration in the early activation of a subcortical mechanism that biases newborns to orient to relevant social visual stimuli (Johnson, 2005). The hypothesis was originally formulated in the context

\section{REFERENCES}

Aguiar, A., and Baillargeon, R. (1999). 2.5-Month-Old Infants' Reasoning about when objects should and should not be occluded. Cogn. Psychol. 39, 116-157. doi: 10.1006/cogp.1999.0717

Aguiar, A., and Baillargeon, R. (2002). Developments in young infants' reasoning about occluded objects. Cogn. Psychol. 45, 267-336. doi: 10.1016/s00100285(02)00005-1

Andrew, R. J. (1991). Neural and Behavioural Plasticity: The Use of the Domestic Chick as a Model. New York, NY: Oxford University Press.

Arya, G. H., Magwire, M. M., Huang, W., Serrano-Negron, Y. L., Mackay, T. F. C., and Anholt, R. R. H. (2015). The genetic basis for variation in olfactory behavior in Drosophila melanogaster. Chem. Senses 40,1-11. doi: 10.1093/chemse/bjv001

Aslin, R. N. (2007). What's in a look? Dev. Sci. 10, 48-53. doi: 10.1111/j.1467-7687. 2007.00563.x

Baillargeon, R., Spelke, E. S., and Wasserman, S. (1985). Object permanence in five-month-old infants. Cognition 20, 191-208.

Barlow, G. (2000). Cichlid Fishes: Nature's Grand Experiment in Evolution. Harper Collins

Bartocci, M., Winberg, J., Ruggiero, C., Bergqvist, L. L., Serra, G., and Lagercrantz, H. (2000). Activation of olfactory cortex in newborn infants after odor stimulation: a functional near-infrared spectroscopy study. Pediatr. Res. 48, 18-23. doi: 10.1203/00006450-200007000-00006

Bateson, P. (1991). Is imprinting such a special case? Philos. Trans. Biol. Sci. 329, 125-131. doi: 10.1093/acprof:oso/9780198521983.003.0004

Blakemore, C., and Van Sluyters, R. C. (1975). Innate and environmental factors in the development of the kitten's visual cortex. J. Physiol. 248, 663-716. doi: 10. 1113/jphysiol.1975.sp010995

Brannon, E. M., Abbott, S., and Lutz, D. J. (2004). Number bias for the discrimination of large visual sets in infancy. Cognition 93, 59-68. doi: 10. 1016/j.cognition.2004.01.004

Brown, A. A., Spetch, M. L., and Hurd, P. L. (2007). Growing in circles: rearing environment alters spatial navigation in fish. Psychol. Sci. 18, 569-573. doi: 10. 1037/e603982013-031

Brugger, P. (2015). Chicks with a number sense. Science 347, 477-478. doi: 10. 1126/science.aaa4854

Cheour, M., Imada, T., Taulu, S., Ahonen, A., Salonen, J., and Kuhl, P. (2004). Magnetoencephalography is feasible for infant assessment of auditory discrimination. Exp. Neurol. 190, 44-51. doi: 10.1016/j.expneurol.2004.06.030

Chiandetti, C., Spelke, E. S., and Vallortigara, G. (2014). Inexperienced newborn chicks use geometry to spontaneously reorient to an artificial social partner. Dev. Sci. 18, 972-978. doi: 10.1111/desc.12277

Chiandetti, C., and Vallortigara, G. (2011a). Chicks like consonant music. Psychol. Sci. 22, 1270-1273. doi: 10.1177/0956797611418244

Chiandetti, C., and Vallortigara, G. (2011b). Intuitive physical reasoning about occluded objects by inexperienced chicks. Proc. R. Soc. B Biol. Sci. 278, 2621-2627. doi: 10.1098/rspb.2010.2381

Chiandetti, C., and Vallortigara, G. (2008). Is there an innate geometric module? Effects of experience with angular geometric cues on spatial re-orientation based on the shape of the environment. Anim. Cogn. 11, 139-146. doi: 10. 1007/s10071-007-0099-y of studies on chicks spontaneous preferences (Morton and Johnson, 1991; Rosa Salva et al., 2011) and recent work with this animal model inspired recent attempts to develop early bio-markers of these neurodevelopmental disorders (Di Giorgio et al., 2014; Johnson, 2014; Rosa Salva et al., 2015) and the study of the underlying molecular mechanisms (Nishigori et al., 2013).

\section{ACKNOWLEDGMENT}

This work is supported by an ERC Advanced Grant PREMESOR ERC-2011-ADG_20110406 awarded to GV.

Cole, S., Hainsworth, F. R., Kamil, A. C., Mercier, T., and Wolf, L. L. (1982). Spatial learning as an adaptation in hummingbirds. Science 217, 655-657. doi: 10 $1126 /$ science. 217.4560 .655

Coubart, A., Izard, V., Spelke, E. S., Marie, J., and Streri, A. (2014). Dissociation between small and large numerosities in newborn infants. Dev. Sci. 17, 11-22. doi: $10.1111 /$ desc. 12108

Datta, S. R., Vasconcelos, M. L., Ruta, V., Luo, S., Wong, A., Demir, E., et al. (2008) The Drosophila pheromone cVA activates a sexually dimorphic neural circuit. Nature 452, 473-477. doi: 10.1038/nature06808

Di Giorgio, E., Lunghi, M., Vallortigara, G., and Simion, F. (2014). Perceptual cues that trigger perception of animacy at birth: the case of self-propulsion, in Proceedings of the 19th International Conference on Infant Studies (ICIS), July 2-4, Berlin, Germany.

Douaud, M., Feve, K., Pituello, F., Gourichon, D., Boitard, S., Leguern, E., et al. (2011). Epilepsy caused by an abnormal alternative splicing with dosage effect of the SV2A gene in a chicken model. PLoS One 6:e26932. doi: 10.1371/journal. pone.0026932

Drucker, C. B., and Brannon, E. M. (2015). Commentary on: "Number-space mapping in the newborn chick resembles humans' mental number line”. Front. Psychol. 6:352. doi: 10.3389/fpsyg.2015.00352

Dubnau, J. ed. (2014). Behavioral Genetics of the Fly (Drosophila melanogaster). Cambridge, UK: Cambridge University Press. doi: 10.1017/cbo97805119 20585

Dulac, C., and Wagner, S. (2006). Genetic analysis of brain circuits underlying pheromone signaling. Annu. Rev. Genet. 40, 449-467. doi: 10.1146/annurev. genet.39.073003.093937

Dunlap, A. S., and Stephens, D. W. (2009). Components of change in the evolution of learning and unlearned preference. Proc. R. Soc. B. Biol. Sci. 276, 3201-8.

Dunlap, A. S., and Stephens, D. W. (2014). Experimental evolution of prepared learning. Proc. Natl. Acad. Sci. U S A 111, 11750-11755. doi: 10.1073/pnas. 1404176111

Dweck, H. K. M., Ebrahim, S. A. M., Kromann, S., Bown, D., Hillbur, Y., Sachse, S., et al. (2013). Olfactory preference for egg laying on citrus substrates in Drosophila. Curr. Biol. 23, 2472-2480. doi: 10.1016/j.cub.2013. 10.047

Fantz, R. L. (1957). Form preferences in newly hatched chicks. J. Comp. Physiol. Psychol. 50, 422-430. doi: 10.1037/h0044973

Fantz, R. L. (1963). Pattern vision in newborn infants. Science 140, 296-297. doi: $10.1126 /$ science. 140.3564 .296

Gagliardi, G. J., Gallup, G. G., and Boren, J. L. (1976). Effect of different pupil to eye size ratios on tonic immobility in chickens. Bull. Psychon. Soc. 8, 58-60. doi: 10.3758/bf03337075

Gallup, G. G., and Nash, R. F. (1971). Tonic immobility as a reaction to predation: artificial eyes as a fear stimulus for chickens. Psychon. Sci. 23, 79-80. doi: 10. 3758/bf03336016

Gandhi, T., Kalia, A., Ganesh, S., and Sinha, P. (2015). Immediate susceptibility to visual illusions after sight onset. Curr. Biol. 25, R358-R359. doi: 10.1016/j.cub. 2015.03.005

Gao, S., Takemura, S. Y., Ting, C. Y., Huang, S., Lu, Z., Luan, H., et al. (2008). The neural substrate of spectral preference in Drosophila. Neuron 60, 328-342. doi: 10.1016/j.neuron.2008.08.010 
Garcia, J., and Koelling, R. A. (1966). Relation of cue to consequence in avoidance learning. Psychon. Sci. 4, 123-124. doi: 10.3758/bf03 342209

Gill, F. B., and Wolf, L. L. (1977). Nonrandom foraging by sunbirds in a patchy environment. Ecology 58, 1284-1296. doi: 10.2307/1935081

Giurfa, M., Nunez, J., Chittka, L., and Menzel, R. (1995). Colour preferences of flower-naive honeybees. J. Comp. Physiol. A 177, 247-259. doi: 10. 1007/bf00192415

Gong, Z. (2012). Innate preference in Drosophila melanogaster. Sci. China Life Sci. 55, 8-14. doi: 10.1007/s11427-012-4271-5

Goren, C. C., Sarty, M., and Wu, P. Y. (1975). Visual following and pattern discrimination of face-like stimuli by newborn infants. Pediatrics 56, 544-549.

Gottlieb (1965). Imprinting in relation to parental and species identification by avian neonates. J. Comp. Physiol. Psychol. 59, 345-356. doi: 10.1037/h0022045

Gottlieb, G. (1968). Prenatal behavior of birds. Q. Rev. Biol. 43, 148. doi: 10. $1086 / 405726$

Graven, S. N., and Browne, J. V. (2008). Sensory development in the fetus, neonate and infant: introduction and overview. Newborn Infant Nurs. Rev. 8, 169-172. doi: 10.1053/j.nainr.2008.10.007

Gumbert, A. (2000). Color choices by bumble bees (Bombus terrestris): innate preferences and generalization after learning. Behav. Ecol. Sociobiol. 48, 36-43. doi: $10.1007 / \mathrm{s} 002650000213$

Hahn, N., Geurten, B., Gurvich, A., Piepenbrock, D., Kstner, A., Zanini, D., et al. (2013). Monogenic heritable autism gene neuroligin impacts Drosophila social behaviour. Behav. Brain Res. 252, 450-457. doi: 10.1016/j.bbr.2013. 06.020

Harshaw, C., and Lickliter, R. (2011). Biased embryos: prenatal experience alters the postnatal malleability of auditory preferences in bobwhite quail. Dev. Psychobiol. 53, 291-302. doi: 10.1002/dev.20521

Haun, D. B. M., Jordan, F. M., Vallortigara, G., and Clayton, N. S. (2010). Origins of spatial, temporal and numerical cognition: insights from comparative psychology. Trends Cogn. Sci. 14, 552-60. doi: 10.1016/j.tics.2010. 09.006

Hepper, P. G., and Shahidullah, B. S. (1994). Development of fetal hearing. Arch. Dis. Child.71, F81-F87. doi: 10.1136/fn.71.2.f81

Hess, E. H. (1973). Imprinting. New York: Van Nostrand Reinhold Company.

Hess, E. H. (1956). Natural preferences of chicks and ducklings for objects of different colors. Psychol. Rep. 2, 477-483. doi: 10.2466/pr0.2.7.477-483

Hess, E. H., and Gogel, W. C. (1954). Natural preferences of the chick for objects of different colors. J. Psychol. 38, 483-493. doi: 10.1080/00223980.1954.9712955

De Hevia, M. D., Izard, V., Coubart, A., Spelke, E. S., and Streri, A. (2014). Representations of space, time and number in neonates. Proc. Natl. Acad. Sci. U S A 111, 4809-4813.

De Hevia, M. D., and Spelke, E. S. (2010). Number-space mapping in human infants. Psychol. Sci. 21, 653-660. doi: 10.1177/0956797610366091

Hossie, T. J., and Sherratt, T. N. (2013). Defensive posture and eyespots deter avian predators from attacking caterpillar models. Anim. Behav. 86, 383-389. doi: 10. 1016/j.anbehav.2013.05.029

Izard, V., Sann, C., Spelke, E. S., and Streri, A. (2009). Newborn infants perceive abstract numbers. Proc. Natl. Acad. Sci. U S A 106, 10382-10385. doi: 10 1073/pnas.0812142106

Janzen, D. H., Hallwachs, W., and Burns, J. M. (2010). A tropical horde of counterfeit predator eyes. Proc. Natl. Acad. Sci. US A 107, 11659-11665. doi: 10. 1073/pnas.0912122107

Johansson, G. (1973). Visual perception of biological motion and a model for its analysis. Percept. Psychophys. 14, 201-211.

Johnson, M. H. (2014). Autism: demise of the innate social orienting hypothesis. Curr. Biol. 24, R30-R31. doi: 10.1016/j.cub.2013.11.021

Johnson, M. H. (2005). Subcortical face processing. Nat. Rev. Neurosci. 6, 766-774. doi: $10.1038 / \mathrm{nrn} 1766$

Johnson, M. H., Dziurawiec, S., Ellis, H., and Morton, J. (1991). Newborns' preferential tracking of face-like stimuli and its subsequent decline. Cognition 40, 1-19. doi: 10.1016/0010-0277(91)90045-6

Johnson, M. H., and Horn, G. (1988). Development of filial preferences in darkreared chicks. Anim. Behav. 36, 675-683. doi: 10.1016/s0003-3472(88)80150-7

Johnson, M. H., Senju, A., and Tomalski, P. (2014). The two-process theory of face processing: modifications based on two decades of data from infants and adults. Neurosci. Biobehav. Rev. 50, 169-179. doi: 10.1016/j.neubiorev.2014. 10.009
Kamil, A. C. (1978). Systematic foraging by a nectar-feeding bird, the amakihi (Loxops virens). J. Comp. Physiol. Psychol. 92, 388-396. doi: 10.1037/h0077479

Kovach, J. (1990). Nonspecific imprintability of quail to colors: response to artificial selection. Behav. Genet. 20, 91-96.

Kovach, J. K. (1983). Constitutional biases in early perceptual learning: I. Preferences between colors, patterns and composite stimuli of colors and patterns in genetically manipulated and imprinted quail chicks (C. coturnix japonica). J. Comp. Psychol. 97, 226-239. doi: 10.1037/0735-7036.97.3.226

Krechevsky, I. (1933). Hereditary Nature of "Hypotheses". J. Comp. Psychol. 16, 99-116. doi: 10.1037/h0072223

Krishnan, B., Dryer, S. E., and Hardin, P. E. (1999). Circadian rhythms in olfactory responses of Drosophila melanogaster. Nature 400, 375-378.

Krishnan, P., Chatterjee, A., Tanoue, S., and Hardin, P. E. (2008). Spike amplitude of single-unit responses in antennal sensillae is controlled by the Drosophila circadian clock. Curr. Biol. 18, 803-807. doi: 10.1016/j.cub.2008.04.060

Lecanuet, J. P., and Schaal, B. (1996). Fetal sensory competencies. Eur. J. Obstet. Gynecol. Reprod. Biol. 68, 1-23. doi: 10.1016/0301-2115(96)02509-2

Lee, S. A., and Vallortigara, G. (2015). Bumblebees spontaneously map location of conspecific using geometry and features. Learn. Motiv. 50, 32-38. doi: 10 1016/j.lmot.2014.10.004

Lee, S. A., Vallortigara, G., Fiore, M., Spelke, E. S., and Sovrano, V. A. (2013) Navigation by environmental geometry: the use of zebrafish as a model. J. Exp. Biol. 216, 3693-3699. doi: 10.1242/jeb.088625

Leslie, A. M., and Keeble, S. (1987). Do six-month-old infants perceive causality? Cognition 25, 265-288. doi: 10.1016/s0010-0277(87)80006-9

Li, Q., and Liberles, S. D. (2015). Aversion and attraction through olfaction. Curr. Biol. 25, R120-R129. doi: 10.1016/j.cub.2014.11.044

Libertus, M. E., and Brannon, E. M. (2010). Stable individual differences in number discrimination in infancy. Dev. Sci. 13, 900-906. doi: 10.1111/j.1467-7687.2009. 00948.x

Libertus, M. E., Pruitt, L. B., Woldorff, M. G., and Brannon, E. M. (2009). Induced alpha-band oscillations reflect ratio-dependent number discrimination in the infant brain. J. Cogn. Neurosci. 21, 2398-2406. doi: 10.1162/jocn.2008.21162

Lickliter, R., and Stoumbos, J. (1991). Enhanced prenatal auditory experience facilitates species-specific visual responsiveness in bobwhite quail chicks (Colinus virginianus). J. Comp. Psychol. 105, 89-94. doi: 10.1037/0735-7036. 105.1.89

LoLordo, V. M. (1979). Constraints on learning. Anim. Learn. Surv. Anal. 19, 473-504. doi: 10.4159/harvard.9780674331495.c9

Lunau, K., and Maier, E. J. (1995). Innate colour preferences of flower visitors. J. Comp. Physiol. A 177, 1-19. doi: 10.1007/bf00243394

Luo, Y., and Baillargeon, R. (2005). Can a self-propelled box have a goal?-psychological reasoning in 5-month-old infants. Psychol. Sci. 16, 601-608. doi: 10.1111/j.1467-9280.2005.01582.x

Manoli, D. S., Meissner, G. W., and Baker, B. S. (2006). Blueprints for behavior: genetic specification of neural circuitry for innate behaviors. Trends Neurosci. 29, 444-451. doi: 10.1016/j.tins.2006.06.006

Mascalzoni, E., Regolin, L., and Vallortigara, G. (2010). Innate sensitivity for selfpropelled causal agency in newly hatched chicks. Proc. Natl. Acad. Sci. U S A 107, 4483-4485. doi: 10.1073/pnas.0908792107

Mascalzoni, E., Regolin, L., Vallortigara, G., and Simion, F. (2013). The cradle of causal reasoning: newborns' preference for physical causality. Dev. Sci. 16, 327-335. doi: 10.1111/desc. 12018

Mery, F., and Kawecki, T. J. (2002). Experimental evolution of learning ability in fruit flies. Proc. Natl. Acad. Sci. U S A 99, 14274-14279. doi: 10.1073/pnas. 222371199

Mileusnic, R., and Rose, S. (2010). The chick as a model for the study of the cellular mechanisms and potential therapies for Alzheimer's disease. Int. J. Alzheimers. Dis. 2010, 1-10. doi: 10.4061/2010/180734

Min, S., Ai, M., and Shin, S. A., and Suh, G. S. B. (2013). Dedicated olfactory neurons mediating attraction behavior to ammonia and amines in Drosophila. Proc. Natl. Acad. Sci. U S A 110, E1321-E1329. doi: 10.1073/pnas.1215 680110

Morton, J., and Johnson, M. H. (1991). CONSPEC and CONLERN: a two-process theory of infant face recognition. Psychol. Rev. 98, 164-181. doi: 10.1037/0033295x.98.2.164

Newcombe, N., Huttenlocher, J., and Learmonth, A. (1999). Infants' coding of location in continuous space. Infant Behav. Dev. 22, 483-510. doi: 10 1016/s0163-6383(00)00011-4 
Nishigori, H., Kagami, K., Takahashi, A., Tezuka, Y., Sanbe, A., and Nishigori, H. (2013). Impaired social behavior in chicks exposed to sodium valproate during the last week of embryogenesis. Psychopharmacology (Berl). 227, 393-402. doi: 10.1007/s00213-013-2979-y

Oliveira, R. F. (2013). Mind the fish: zebrafish as a model in cognitive social neuroscience. Front. Neural Circuits 7:131. doi: 10.3389/fncir.2013.00131

Perani, D., Saccuman, M. C., Scifo, P., Spada, D., Andreolli, G., Rovelli, R., et al. (2010). Functional specializations for music processing in the human newborn brain. Proc. Natl. Acad. Sci. U S A 107, 4758-4763. doi: 10.1073/pnas. 0909074107

Potrich, D., Stancher, G., and Sovrano, V. A., Vallortigara, G. (2015). Quantity discrimination in zebrafish (Danio rerio). J. Comp. Psychol. 129, 388-393. doi: $10.1037 /$ com0000012

Regolin, L., Vallortigara, G., and Zanforlin, M. (1995). Object and spatial representations in detour problems by chicks. Anim. Behav. 49, 195-199. doi: 10.1016/0003-3472(95)80167-7

Reiter, L. T., Potocki, L., Chien, S., Gribskov, M., and Bier, E. (2001). A systematic analysis of human disease-associated gene sequences in Drosophila melanogaster. Genome Res. 11, 1114-1125. doi: 10.1101/gr. 169101

Rollmann, S. M., Wang, P., Date, P., West, S. A., Mackay, T. F. C., and Anholt, R. R. H. (2010). Odorant receptor polymorphisms and natural variation in olfactory behavior in Drosophila melanogaster. Genetics 186, 687-697. doi: 10. 1534/genetics.110.119446

Ronderos, D. S., Lin, C.-C., Potter, C. J., and Smith, D. P. (2014). Farnesoldetecting olfactory neurons in Drosophila. J. Neurosci. 34, 3959-3968. doi: 10. 1523/jneurosci.4582-13.2014

Root, C. M., Ko, K. I., Jafari, A., and Wang, J. W. (2011). Presynaptic facilitation by neuropeptide signaling mediates odor-driven food search. Cell 145, 133-144. doi: 10.1016/j.cell.2011.02.008

Rosa Salva, O., Farroni, T., Regolin, L., Vallortigara, G., and Johnson, M. H. (2011). The evolution of social orienting: evidence from chicks (Gallus gallus) and human newborns. PLoS One 6:e18802. doi: 10.1371/journal.pone. 0018802

Rosa Salva, O., Mayer, U., and Vallortigara, G. (2015). Roots of a social brain: developmental models of emerging animacy-detection mechanisms. Neurosci. Biobehav. Rev. 50, 150-168. doi: 10.1016/j.neubiorev.2014.12.015

Rosa-Salva, O., Regolin, L., and Vallortigara, G. (2010). Faces are special for newly hatched chicks: evidence for inborn domain-specific mechanisms underlying spontaneous preferences for face-like stimuli. Dev. Sci. 13, 565-77. doi: 10. $1111 /$ j.1467-7687.2009.00914.x

Ruebenbauer, A., Schlyter, F., Hansson, B. S., Löfstedt, C., and Larsson, M. C. (2008). Genetic variability and robustness of host odor preference in Drosophila melanogaster. Curr. Biol. 18, 1438-1443. doi: 10.1016/j.cub.2008.08.062

Rugani, R., Fontanari, L., Simoni, E., Regolin, L., and Vallortigara, G. (2009). Arithmetic in newborn chicks. Proc. Biol. Sci. 276, 2451-2460. doi: 10. 1098/rspb.2009.0044

Rugani, R., Regolin, L., and Vallortigara, G. (2010). Imprinted numbers: newborn chicks' sensitivity to number vs. continuous extent of objects they have been reared with. Dev. Sci. 13, 790-797. doi: 10.1111/j.1467-7687.2009. 00936.x

Rugani, R., Rosa Salva, O., Regolin, L., and Vallortigara, G. (2015a). Brain asymmetry modulates perception of biological motion in newborn chicks (Gallus gallus). Behav. Brain Res. 290, 1-7. doi: 10.1016/j.bbr.2015. 04.032

Rugani, R., Vallortigara, G., Priftis, K., and Regolin, L. (2015b). Number-space mapping in the newborn chick resembles humans' mental number line. Science 347, 534-536. doi: 10.1126/science.aaa1379

Rugani, R., Vallortigara, G., and Regolin, L. (2014). From small to large: numerical discrimination by young domestic chicks (Gallus gallus). J. Comp. Psychol. 128, 163-171. doi: 10.1037/a0034513

Ruta, V., Datta, S. R., Vasconcelos, M. L., Freeland, J., Looger, L. L., and Axel, R. (2010). A dimorphic pheromone circuit in Drosophila from sensory input to descending output. Nature 468, 686-690. doi: 10.1038/nature09554

Schaal, B., Marlier, L., and Soussignan, R. (2000). Human foetuses learn odours from their pregnant mother's diet. Chem. Senses 25, 729-737. doi: 10. 1093/chemse/25.6.729

Schaefer, H., and Hess, H. (1951). Color preferences in imprinting objects. Z. Tierpsychol. 16, 161-172. doi: 10.1111/j.1439-0310.1959.tb02051.x
Schlötterer, C., Kofler, R., Versace, E., Tobler, R., and Franssen, S. U. (2015). Combining experimental evolution with next-generation sequencing: a powerful tool to study adaptation from standing genetic variation. Heredity (Edinb). 114, 431-440. doi: 10.1038/hdy.2014.86

Schulman, A. H., Hale, B. E., and Graves, H. (1970). Visual stimulus characteristics for initial approach response in chicks (Gallus domesticus). Anim. Behav. 18, 461-466. doi: 10.1016/0003-3472(70)90040-0

Semmelhack, J. L., and Wang, J. W. (2009). Select Drosophila glomeruli mediate innate olfactory attraction and aversion. Nature 459, 218-223. doi: 10. 1038/nature07983

Simion, F., Regolin, L., and Bulf, H. (2008). A predisposition for biological motion in the newborn baby. Proc. Natl. Acad. Sci. U S A 105, 809-813. doi: 10. 1073/pnas.0707021105

Spelke, E. S., Lee, S. A., and Izard, V. (2010). Beyond core knowledge: natural geometry. Cogn. Sci. 34, 863-884. doi: 10.1111/j.1551-6709.2010.01110.x

Spelke, E. S. (1998). Nativism, empiricism and the origins of knowledge. Infant Behav. Dev. 21, 181-200. doi: 10.1016/s0163-6383(98)90002-9

Spelke, E. S., and Kinzler, K. D. (2007). Core knowledge. Dev. Sci. 10, 89-96. doi: 10.1111/j.1467-7687.2007.00569.x

Spelke, E. S., and Newport, E. L. (1998). "Nativism, empiricism and the development of knowledge," in Handbook of Child Psychology: Vol. 1. Theoretical Models of Human Development, eds W. Damon and R. Lerner (New York, NY: Wiley), 275-340.

Starr, A., Libertus, M. E., and Brannon, E. M. (2013). Number sense in infancy predicts mathematical abilities in childhood. Proc. Natl. Acad. Sci. U S A 110 18116-20. doi: 10.1073/pnas.1302751110

Stevens, M., Hardman, C. J., and Stubbins, C. L. (2008). Conspicuousness, not eye mimicry, makes "eyespots" effective antipredator signals. Behav. Ecol. 19, 525-531. doi: 10.1093/beheco/arm162

Streri, A., de Hevia, M., Izard, V., and Coubart, A. (2013). What do we know about neonatal cognition? Behav. Sci. Basel 3, 154-169. doi: 10.3390/bs30 10154

Suh, G. S. B., Wong, A. M., Hergarden, A. C., Wang, J. W., Simon, A. F., Benzer, S., et al. (2004). A single population of olfactory sensory neurons mediates an innate avoidance behaviour in Drosophila. Nature 431, 854-859. doi: 10.3410/f. 1021993.251709

Tommasi, L., Chiandetti, C., Pecchia, T., Sovrano, V. A., and Vallortigara, G. (2012). From natural geometry to spatial cognition. Neurosci. Biobehav. Rev. 36, 799-824. doi: 10.1016/j.neubiorev.2011.12.007

Trainor, L. J., Tsang, C. D., and Cheung, V. H. W. (2002). Preference for sensory consonance in 2- and 4-month-old infants. Music Percept. 20, 187-194. doi: 10. 1525/mp.2002.20.2.187

Turati, C., Simion, F., Milani, I., and Umiltà, C. (2002). Newborns' preference for faces: what is crucial? Dev. Psychol. 38, 875-882. doi: 10.1002/97804706 93735.ch9

Vallortigara, G. (2012a). "Aristotle and the chicken: Animacy and the origins of beliefs," in The Theory of Evolution and its Impact, ed. A. Fasolo (New York, NY: Springer International Publishing), 189-200.

Vallortigara, G. (2012b). Core knowledge of object, number and geometry: a comparative and neural approach. Cogn. Neuropsychol. 29, 213-236. doi: 10. $1080 / 02643294.2012 .654772$

Vallortigara, G. (2015). "Foundations of number and space representations in precocial species," in Evolutionary Origins and Early Development of Number Processing, Mathematical Cognition and Learning, Vol. 1, (London: Elsevier), 35-66.

Vallortigara, G., and Regolin, L. (2006). Gravity bias in the interpretation of biological motion by inexperienced chicks. Curr. Biol. 16, 279-280. doi: 10. 1016/j.cub.2006.03.052

Vallortigara, G., Regolin, L., and Marconato, F. (2005). Visually inexperienced chicks exhibit spontaneous preference for biological motion patterns. PLoS Biol. 3:e208. doi: 10.1371/journal.pbio.0030208

Vallortigara, G., Rogers, L. J., and Bisazza, A. (1999). Possible evolutionary origins of cognitive brain lateralization. Brain Res. Rev. 30, 164-175. doi: 10. 1016/s0165-0173(99)00012-0

Vallortigara, G., Sovrano, V. A., and Chiandetti, C. (2009). Doing Socrates experiment right: controlled rearing studies of geometrical knowledge in animals. Curr. Opin. Neurobiol. 19, 20-26. doi: 10.1016/j.conb.2009.02.002

Versace, E. (2015). Experimental evolution, behavior and genetics: associative learning as a case study. Curr. Zool. 61, 226-241. 
Versace, E., and Reisenberger, J. (2015). Large-scale assessment of olfactory preferences and learning in Drosophila melanogaster: behavioural and genetic components. PeerJ 3:e1214. doi: 10.7717/peerj.1214

Van der Voet, M., Nijhof, B., Oortveld, M. A. W., and Schenck, A. (2014). Drosophila models of early onset cognitive disorders and their clinical applications. Neurosci. Biobehav. Rev. 46, 326-342. doi: 10.1016/j.neubiorev. 2014.01.013

Walk, R. D., and Gibson, E. J. (1961). A comparative and analytical study of visual depth perception. Psychol. Monogr. Gen. Appl. 75, 1-44. doi: 10.1037/h00 93827

Walk, R. D., Gibson, E. J., and Tighe, T. J. (1957). Behavior of light-and darkreared rats on a visual cliff. Science 126, 80-81. doi: 10.1126/science.126. 3263.80-a

Wilkinson, A., Mandl, I., Bugnyar, T., and Huber, L. (2010). Gaze following in the red-footed tortoise (Geochelone carbonaria). Anim. Cogn. 13, 765-769. doi: 10. 1007/s10071-010-0320-2

Wong, J. W. Y., Meunier, J., and Kölliker, M. (2013). The evolution of parental care in insects: the roles of ecology, life history and the social environment. Ecol. Entomol. 38, 123-137. doi: 10.1111/een.12000

Wood, S. M. W., and Wood, J. N. (2015). A chicken model for studying the emergence of invariant object recognition. Front. Neural Circuits 9:7. doi: 10. 3389/fncir.2015.00007
$\mathrm{Xu}$, F. (2003). Numerosity discrimination in infants: evidence for two systems of representations. Cognition 89, 15-25. doi: 10.1016/s0010-0277(03) 00050-7

Xu, F., Spelke, E. S., and Goddard, S. (2005). Number sense in human infants. Dev. Sci. 8, 88-101. doi: 10.1111/j.1467-7687.2005.00395.x

Yamaguchi, S., Desplan, C., and Heisenberg, M. (2010). Contribution of photoreceptor subtypes to spectral wavelength preference in Drosophila. Proc. Natl. Acad. Sci. U S A 107, 5634-5639. doi: 10.1073/pnas.0809398107

Zanforlin, M., and Vallortigara, G. (1985). Form preferences and stimulus generalization in domestic chicks. Boll. Zool. 52, 231-238. doi: 10 $1080 / 11250008509440523$

Conflict of Interest Statement: The authors declare that the research was conducted in the absence of any commercial or financial relationships that could be construed as a potential conflict of interest.

Copyright (c) 2015 Versace and Vallortigara. This is an open-access article distributed under the terms of the Creative Commons Attribution License (CC BY). The use, distribution and reproduction in other forums is permitted, provided the original author(s) or licensor are credited and that the original publication in this journal is cited, in accordance with accepted academic practice. No use, distribution or reproduction is permitted which does not comply with these terms. 